\title{
Por que é tão difícil inovar nas práticas de ensino? Um modelo de formação universitária centrado na saúde pedagógica*
}

Why is it so difficult to innovate in teaching practices? A model of teaching development focused on pedagogic health

¿Por qué es tan difícil innovar en las prácticas de enseñanza?

Un modelo de formación universitaria enfocado en

la salud pedagógica

\section{Joana Guilares de Aguiar}

- Professora adjunta do Departamento de Química Inorgânica, do Instituto de Química da Universidade Federal Fluminense (UFF)

- Pós-doutora pela Faculdade de Educação da Universidade de São Paulo (FE-USP)

- Mestre e doutora em Ensino de Química pelo Programa Interunidades em Ensino de Ciências da USP

- Especialista em Docência no Ensino Superior pela Universidade Nove de Julho (Uninove)

- Graduada em Química pela Universidade Estadual de Campinas (Unicamp)

- E-mail: joana_aguiar@id.uff.br

* A autora agradece aos professores Maurício Pietrocola (FE-USP), Paulo Rogério Correia (EACH-USP) e Ian Kinchin (University of Surrey, UK) pelas valiosas discussões que culminaram neste trabalho. E também à Fundação de Amparo à Pesquisa do Estado de São Paulo (Fapesp) pelo financiamento (Processo Fapesp \#2018/21857-0). 


\title{
Resumo
}

O ambiente de ensino universitário é dinâmico e complexo. Porém, as abordagens didáticas continuam sendo prevalentemente conservadoras, tradicionais e focadas na transmissão de conteúdo disciplinar. Apesar da necessidade de inovação didático-pedagógica, por que, na prática, ainda parece ser tão difícil inovar em nossas práticas de ensino? Este artigo tem o objetivo de explorar uma visão humanista para abordar o problema, focada na promoção da saúde pedagógica na universidade como forma de potencializar esforços individuais e institucionais para uma educação inovadora.

\section{PALAVRAS-CHAVE: DESENVOLVIMENTO PEDAGÓGICO • ENSINO SUPERIOR • INOVAÇÃO DIDÁTICA • SAÚDE PEDAGÓGICA • UNIVERSIDADE.}

\begin{abstract}
Teaching environment at university is dynamic and complex. However, teaching approaches continue to be predominantly conservative, traditional and focused on the transmission of disciplinary content. Despite the need for teaching innovation, why, practically speaking, is it so difficult to innovate in our teaching practices? This paper aims to explore a humanist approach to address the problem, focused on promoting pedagogic health at the university as a way to enhance individual and institutional efforts for innovative education.
\end{abstract}

\section{KEYWORDS: TEACHING DEVELOPMENT • HIGHER EDUCATION • TEACHING INNOVATION • PEDAGOGIC HEALTH • UNIVERSITY.}

\section{Resumen}

El ambiente de enseñanza universitaria es dinámico y complejo. Sin embargo, los enfoques didácticos continúan siendo predominantemente conservadores, tradicionales y centrados en la transmisión de contenido disciplinar. A pesar de la necesidad de innovación didáctico-pedagógica, ¿por qué, en la práctica, todavía parece tan difícil innovar en nuestras prácticas de enseñanza? Este artículo tiene como objetivo explorar una visión humanista para abordar el problema, centrada en promover la salud pedagógica en la universidad como una forma de mejorar los esfuerzos individuales e institucionales para una educación innovadora.

\section{PALABRAS CLAVES: DESARROLLO PEDAGÓGICO • EDUCACIÓN SUPERIOR • INNOVACIÓN DIDÁCTICA • SALUD PEDAGÓGICA • UNIVERSIDAD.}



problemas presentes e futuros em uma sociedade cada vez mais dinâmica e complexa (UNESCO, 2005).

Apesar disso, as abordagens de ensino na universidade continuam sendo prevalentemente conservadoras e tradicionais, em que a transmissão do conteúdo é vista como dominante. 0 aluno é reduzido a um sujeito destinado a receber passivamente o conhecimento. 0 docente, ao adotar práticas centradas "naquilo que ele já sabe e o que funciona para ele", seleciona a informação que considera essencial para transmitir sua visão de ensino e, seletivamente, ignora o restante dos conteúdos e valores. 0 impacto em longo prazo dessa abordagem pode ser visto na formação do aluno estruturada como uma cadeia linear, a qual reforça a memorização em vez da aprendizagem profunda e significativa necessária ao futuro profissional. Para Martin Talbot (2004), a adoção dessa linearidade reforça uma certeza quase que autoritária da existência de uma única rota possível de ensino, não havendo, portanto, espaço para mudanças, criatividade e inovação.

Uma forma de romper com o estilo didático positivista, que comunica um conhecimento fechado, acabado, regido pela racionalidade técnica, é a promoção da inovação didático-pedagógica. Conforme descrito por Elisa Lucarelli (2000, p.63), "as práticas de ensino inovadoras são aquelas que alteram, de algum modo, o sistema unidirecional de relações que caracterizam o ensino conservador e tradicional". Já em Jaume Carbonell (2002, p. 19) a inovação é tida como um "conjunto de intervenções, decisões com certo grau de intencionalidade e sistematização, que visam transformar as atitudes, ideias, culturas, conteúdos, modelos e práticas pedagógicas". Em um estudo mais recente, Maria Isabel Cunha (2018) entende que a inovação pedagógica se materializa pelo reconhecimento de formas alternativas de saberes e experiências, superando típicas dicotomias desse sistema, tais como teoria versus prática, senso comum versus conhecimento científico, mercado versus academia. Em todas essas definições é possível perceber que o foco da inovação em âmbito educacional está no processo, capaz de alterar e transformar as pessoas e o seu meio (FULLAN, 2000).

Nesse contexto, surge uma pergunta importante: "Se a inovação didático-pedagógica é tão importante e inevitável no contexto universitário brasileiro, por que parece ser tão difícil inovar em nossas práticas de ensino?". Obviamente, esta pergunta não é simples de ser respondida. Este artigo tem o objetivo de explorar e propor uma visão holística e humanista para abordar o problema, focada na promoção da saúde pedagógica no ambiente universitário como forma de potencializar os esforços institucionais para uma educação inovadora.

\section{DE QUE TIPO DE INOVAÇÃO ESTAMOS FALANDO?}

0 conceito de inovação na educação é polissêmico, amplo e plural (BATTESTIN; NOGARO, 2016). Primeiramente, é muito importante deixar claro que a inovação didática difere grandemente da inovação corporativa, empresarial ou industrial. Esta última, representa a criação de algo novo, uma ideia, método ou objeto diferente do anterior, sendo frequentemente associada ao avanço da ciência e da tecnologia ou ao empreendedorismo (AUDY; MOROSINI, 2006). A inovação nas práticas de ensino não se resume à mera adoção de um novo método, ideia ou objeto em sala de aula e, sim, requer uma profunda mudança nas relações de significado do que é ensinar e aprender.

Recorrer de modo isolado à introdução de técnicas tidas por inovadoras ou a novos dispositivos, se for desligado ou não suficientemente ancorado numa nova visão de conjunto da formação, em concepções claras acerca das metas de aprendizagem dos alunos e acerca do papel e da influência do docente (estas sim, questões desafiadoras e difíceis) pode não trazer significativas melhorias da qualidade da formação realizada (ESTEVES, 2008, p. 106).

Aparentemente, o constante discurso institucional em prol da estratégia de interação entre dois dos componentes dos sistemas de inovação (as universidades e as empresas) pode justificar, em parte, a confusão entre esses tipos de inovação no contexto universitário (KUNSCH, 2019). 
Uma segunda discussão diz respeito a quem exatamente compete a inovação nas práticas de ensino. Atualmente, existem muitas inovações pedagógicas interessantes sendo promovidas na literatura sobre ensino e aprendizagem no âmbito universitário. No entanto, arriscaria dizer que muitas destas estão fadadas ao fracasso, uma vez que o ambiente (e seus indivíduos) não são particularmente receptivos a elas. Essa relação dialética entre as partes (docentes, alunos, coordenação/direção) e o sistema (ambiente de sala de aula, ambiente acadêmico, a universidade, a sociedade) é complexa e determinante para garantir a adoção e manutenção de práticas pedagógicas mais alinhadas com a atual sociedade do conhecimento.

A maioria das pesquisas sobre inovação didática no ensino superior estão centradas em listar e descrever diferentes métodos, técnicas, estratégias e atividades para ensinar e aprender. Apesar de úteis, essas informações por si só podem não ser suficientes para gerar, no docente, a segurança e autoconfiança necessárias para implementar mudanças em sala de aula. Além disso, algumas dessas "inovações" podem não ser viáveis para todos os contextos educacionais, o que requereria do docente não apenas a capacidade de escolher uma nova metodologia como também saber adaptá-la.

A aparente facilidade com que se apresentam as diferentes práticas inovadoras pode ser uma "armadilha" para os docentes, visto que eles podem deduzir que sua utilização em sala de aula é igualmente trivial. Por exemplo, as metodologias ativas da aprendizagem ${ }^{1}$ (BACICH; MORAN, 2018), tais como a aprendizagem baseada em problemas, a aprendizagem por projetos, o ensino híbrido (blended learning), a instrução por pares (peer instruction), a sala de aula invertida (flipped classroom), vêm sendo amplamente adotadas como práticas inovadoras de ensino. Porém, muitas das dificuldades observadas com a efetiva utilização dessas práticas derivam, pelo menos em parte, do uso inadequado das metodologias escolhidas, da falta de informações sobre o procedimento e consecução das atividades, do não reconhecimento da importância dos fundamentos teóricos que as subsidiam e, principalmente, da ausência de mudança nas relações entre docente-aluno.

Os docentes do ensino superior têm adotado uma postura defensiva [em relação a possíveis mudanças], ao considerar que ensinar é somente uma questão de compromisso com o conhecimento científico válido da sua área, e que aprender é um problema exclusivo do aluno, associado à sua determinação, motivações, capacidades, conhecimentos e competências adquiridos anteriormente (ZABALZA, 2004, p. 105).

Em suma, além de interesse, vontade e motivação para inovar, o docente precisa também "saber como" implementar, na prática, as mudanças necessárias bem como ter meios de lidar com resultados inesperados, principalmente no que tange à aceitação dos alunos a essas mudanças. Além disso, dependendo do nível de regulação imposto pelo departamento/ escola/instituto e do espaço cedido para a negociação das práticas de ensino, essa autonomia individual para criar, mudar, transformar e, por fim, inovar surtirá efeitos em nível local (na sala de aula), mas não em nível institucional.

Na prática, o que se percebe é uma multiplicidade de fatores impeditivos para adoção de práticas inovadoras no ensino superior. De fato, para Paulo S. Garcia (2009) elas passam por fatores pessoais (p. ex., medo, insegurança), profissionais (p. ex., falta de apoio, tempo, treinamento, estímulo) e contextuais (p. ex., falta de recursos financeiros, materiais, legislação atrasada), reforçando a necessidade de comprometimento tanto dos docentes como das lideranças acadêmicas e da instituição na busca por uma cultura de inovação pedagógica (FARIAS, 2006).

\section{O MODELO DA FRAGILIDADE PEDAGÓGICA E SUA CONOTAÇÃO NEGATIVA}

Existem muitas formas de abordar o problema da falta de inovação didático-pedagógica universitária. Para Ian Kinchin e Naomi Winstone (2017), por exemplo, os discursos e tensões conflitantes no sistema educacional resultam em um certo grau de "fragilidade pedagógica", a qual pode ser vista, na prática, quando os docentes percebem as pressões cumulativas das atividades acadêmicas como o principal fator que inibe sua capacidade em mudar suas práticas de ensino. A consequência é,

1 Metodologias ativas de aprendizagem são estratégias pedagógicas complexas que possuem como principal característica a adoção do aluno como agente principal e responsável pelo seu processo de aprendizagem. 0 docente é visto como mediador desse processo à medida que apresenta e conduz as tarefas. 
usualmente, a adoção de uma abordagem pedagógica tradicional, "segura", sustentável e, possivelmente, desatualizada (CLEGG, 2008; BAILEY, 2014).

0 modelo da fragilidade pedagógica (KINCHIN et al., 2016, KINCHIN, 2017; KINCHIN e WINSTONE, 2017; AGUIAR e CORREIA, 2019) coloca em foco elementos-chave que descrevem as atividades acadêmicas e os principais motivos que levam muitos docentes, incluindo os mais experientes, a adotar uma abordagem de ensino conservadora e tradicional. Usualmente, a fragilidade pedagógica ocorre na universidade quando há tensões entre os elementos do ambiente de ensino, ou seja, quando:

- 0 foco do discurso curricular está concentrado nos mecanismos e regulamentos ao invés de teorias e valores profissionais que direcionam nossas perspectivas pessoais;

- A relação entre os conteúdos disciplinares e a prática profissional não está alinhada à natureza da disciplina;

- O caráter da relação ensino-pesquisa é desbalanceado e assimétrico e não está explícito nas práticas pedagógicas;

- Há um baixo grau de percepção quanto à proximidade e influência dos docentes sobre o processo de tomada de decisão que impacta o ensino.

Quando os elementos desse sistema estão em tensão entre si, os docentes tendem a sucumbir ao estresse e ao esgotamento mental. Segundo Mary Carlotto (2002), no exercício profissional da atividade docente encontram-se presentes diversos estressores psicossociais, alguns relacionados à natureza de suas funções, outros relacionados ao contexto institucional e social onde estas são exercidas. Quando persistentes, esses estressores podem levar à síndrome de burnout (HARRISON, 1999), um tipo de estresse vinculado a situações de trabalho, resultante da constante e repetitiva pressão emocional associada com intenso envolvimento com pessoas por longos períodos. A literatura sobre estresse e burnout entre docentes (p. ex., MASLACH, 1982; BYRNE, 1991; CARLOTTO e CÂMARA, 2007; BORINE, WANDERLEY e BASSITT, 2015; JEUNON et al., 2017) demonstra que os índices de felicidade e bem-estar, satisfação com a carreira e o trabalho e aspectos da personalidade devem ser levados em consideração quando buscamos a saúde psicológica necessária para sua atuação nas diferentes atividades acadêmicas.

Em um contexto de estresse e esgotamento, qualquer tipo de proposta de inovação didática tem poucas chances de sucesso, pois, usualmente, são vistas como ameaças à percepção (ilusória) de estabilidade do sistema. Portanto, saber lidar com as adversidades e os fatores de estresse impostos no ambiente acadêmico é parte fundamental para dar início ao combate à fragilidade pedagógica.

\section{A PROMOÇÃO DA SAÚDE PEDAGÓGICA E A UNIVERSIDADE SALUTOGÊNICA}

As pesquisas dos últimos cinco anos apontam a utilidade do panorama teórico-empírico da fragilidade pedagógica como um ponto de partida promissor para o desenvolvimento de docentes universitários em diferentes áreas de conhecimento, tais como geografia (KINCHIN; FRANCIS, 2017), artes e humanidades (KINCHIN; WILEY, 2018), ensino de ciências (CORREIA; AGUIAR; KINCHIN, 2017), psicologia (WINSTONE; KINCHIN, 2017), enfermagem (DERHAM, 2018), engenharia (BEHNEJAD, 2018), química (WHELLIGAN, 2018), política (USHERWOOD, 2018), direito (MASON, 2018) e administração (ADCROFT, 2018). Entretanto, sua conotação negativa, focada nos problemas constantemente relatados pelos docentes, vem dificultando sua aceitação e implementação no ensino superior. Nesse contexto, ao invés de considerar um cenário em que a fragilidade pedagógica deve ser combatida, neste artigo é proposto um contraponto positivo, de promoção da saúde pedagógica.

A salutogênese (ANTONOVSKY, 1987) designa as forças que geram saúde, em oposto à patogênese, ou seja, as influências que causam doenças. A analogia médica vem nos ajudar a descrever uma universidade que deve se preocupar com a saúde física, mental, psicológica, emocional, social e cultural dos seus docentes. A partir deste novo paradigma e, já estabelecendo uma ponte para a esfera educacional (NILSSON et al., 2015), pode-se dizer que o ápice da salutogênese é atingida pelo senso de coerência, ou seja, (i) a existência de explicações que evitam a sensação de caos e a ausência de 
propósito, (ii) a presença de uma sensação de significância, que "faz valer a pena" se engajar nas mudanças propostas e (iii) a capacidade de usar suas habilidades para promover mudanças em âmbito local e global.

Muitos dos atuais modelos de formação continuada não se alinham com essa visão. 0 oferecimento de palestras, oficinas, minicursos apesar de interessante, ainda é muito pouco efetivo quando se trata de obter a abertura necessária para promover um diálogo franco, o senso de coerência e, consequentemente, a salutogênese. Em linhas gerais, a saúde pedagógica deve considerar uma condição em que os elementos do ensino universitário contribuam ativamente para o bem-estar dos docentes, com a finalidade de se construir um ambiente de aprendizado saudável, centrado no ser humano e na complexidade das relações interpessoais. Ajudar os elementos desse sistema a se complementarem e se apoiarem como um todo coerente produzirá um ambiente que exibe saúde pedagógica, no qual as inovações têm maior chance de sucesso.

A seguir, foram reunidas algumas diretrizes de promoção da saúde pedagógica como forma de potencializar os esforços individuais e institucionais em prol de uma cultura inovadora na universidade:

\section{1) É fundamental atenção à qualidade de vida pessoal e profissional do docente.}

A capacidade de inovação no ensino deve considerar uma componente pessoal, relativa aos aspectos de personalidade e felicidade na vida em geral, e uma componente de satisfação profissional. Docentes empáticos, criativos, conscientes, confiantes, felizes em sua vida pessoal e satisfeitos com sua carreira, seu trabalho e com a docência tenderão a ser mais receptivos às mudanças impostas pela alta complexidade do ambiente de ensino ou pelas constantes cobranças externas. Além disso, é preciso promover meios de desenvolvimento da resiliência acadêmica. Esta, de caráter interativo, diz respeito à relativa resistência de um indivíduo às experiências de risco em seu ambiente, na superação das adversidades de maneira saudável. Docentes resilientes são aqueles que possuem um bom desempenho psicológico, capazes de se adaptar, aprender e mudar apesar das vivências negativas no ambiente universitário. Quanto maior a resiliência, maior o repertório de estratégias que o docente possui para lidar com circunstâncias adversas ou estressantes de seu cotidiano. 0 mecanismo de aquisição de resiliência pode ser promovido com situações em que o docente explore novas formas de se comportar, permitindo que ele saia de sua "zona de conforto". Porém, conforme já mencionado, é fundamental acompanhar o seu desempenho psicológico a fim de evitar situações de isolamento, estresse extremo e burnout.

\section{2) É preciso discutir sobre as teorias e os valores que informam o currículo do curso.}

As teorias de ensino e aprendizagem e os valores que sustentam o currículo do curso e, consequentemente, informam a nossa prática pedagógica são implícitas e pouco discutidas e compartilhadas entre os docentes. Normalmente, as reuniões de departamento para tratar sobre questões de ensino estão restritas a designar disciplinas aos seus respectivos docentes, alterar ementas, conteúdos disciplinares, carga horárias, bibliografias, definir horas-aula de laboratório ou estágio. Ou seja, o foco do discurso usualmente está concentrado nos mecanismos e regulamentos, sem que para isso seja feita uma reflexão de como essas alterações impactam a qualidade do ensino e, consequentemente, a formação do egresso. 0 docente que deseja inovar em suas práticas de ensino, diante de tamanho discurso burocrático e quase "autoritário", não vê saída a não ser repetir os mesmos materiais e estratégias do ano anterior. É preciso uma predisposição de gestores e docentes em explicitar e compartilhar suas visões de ensino e experiências de sucesso, estando abertos às mudanças e à adoção de novas práticas, alinhadas aos valores do curso.

\section{3) O ensino e a avaliação devem ser autênticos e estarem alinhados com a natureza da disciplina.}

A autenticidade do ensino (BENNETT; HARPER; HEDBERG, 2002) é atingida quando as atividades e estratégias didáticas levam os alunos a compreender o papel do conteúdo especializado no contexto da futura prática profissional, extrapolando 
a mera transmissão de informações, e isto é totalmente idiossincrático à natureza da disciplina e do curso. Ainda que uma disciplina seja de fundamentos teóricos, esse conhecimento precisa ser articulado de forma que o aluno saiba, ao final da disciplina, resolver problemas. Além disso, se o docente escolhe ensinar o conteúdo teórico por meio da aplicação de atividades práticas, sua avaliação precisa estar alinhada a este mesmo pressuposto de ensino-aprendizagem. Se, durante o ensino, é valorizada a construção e a articulação que o aluno faz com o conhecimento, a avaliação não poderá apenas cobrar fatos, nomes e fórmulas, mas deverá também privilegiar a argumentação, a criticidade, a criatividade e a solução prática do problema. Com isso, tanto ensino como avaliação serão autênticos. Por fim, as universidades devem formar egressos com uma rede de conhecimento rica e altamente articulada, se aproximando do especialista na área. Se os conteúdos disciplinares são ensinados de modo linear e desconectados de suas futuras profissões, dificilmente os alunos saberão resolver problemas complexos impostos nos cenários da vida real (KINCHIN; LYGO-BAKER; HAY, 2008).

\section{4) A relação entre ensino e pesquisa deve orientar a prática pedagógica.}

O ingresso na academia requer habilidades de pesquisa e ensino como pré-requisitos dos acadêmicos, os quais devem ser fundidos ao invés de tomarem papéis separados. É muito comum observarmos atualmente uma distribuição não balanceada de tempo e energia dedicados às atividades de ensino e pesquisa (ROBERTSON e BOND, 2001; HOSEIN, 2017), em que uma maior valorização percebida para um dos lados pode levar ao desequilíbrio que desencadeia desdobramentos assimétricos de recompensas, status, reconhecimento e motivação. Uma tensão similar também é observada com docentes que lecionam no ensino superior e atuam no mercado de trabalho. Possuir diferentes atribuições ao longo da carreira acadêmica não deve se configurar como um problema, mas sim como uma oportunidade para que os docentes informem suas práticas pedagógicas. Situações reais do meio corporativo e/ou resultados de pesquisa recentes da literatura podem (e devem!) atuar como uma fonte de informação que auxilia a escolha de conteúdos disciplinares e estratégias de ensino direcionadas à solução de problemas e pensamento crítico. De forma análoga, discussões produtivas geradas em sala de aula podem ser usadas como "inspiração" para iniciar novas pesquisas na área ou ainda oferecer uma abordagem diferente de enfrentamento do problema.

\section{5) Docentes devem atuar junto às lideranças acadêmicas nas tomadas de decisões que impactam o ensino.}

Nos últimos anos, algumas universidades deslocaram-se para uma administração mais centralizada, com modelos de avaliação da qualidade do ensino com uma visão "comercial" (GOERGEN, 2010), além de rigorosas abordagens de auditoria para medir a eficácia desse ensino prestado aos alunos (vistos aqui como consumidores). Nesse modelo, docentes e alunos são desconsiderados do processo de tomada de decisões que impactam o ensino. A consequência é o desinteresse e o descomprometimento com a qualidade da formação oferecida na universidade. Uma forma de reverter essa situação é a adoção de uma administração descentralizada, que permite maior participação da comunidade acadêmica nas tomadas de decisões e no processo de avaliação da qualidade de ensino (SOUSA SANTOS, 2010), promovendo o senso de coerência. 0 engajamento para mudança e inovação pedagógica depende, em grande parte, desta sensação de pertencimento e alinhamento aos valores institucionais, os quais devem ser partilhados por toda a comunidade acadêmica, ou seja, alunos, docentes, funcionários e lideranças acadêmicas locais (chefes de departamento, coordenadores, colegiados) e globais (gestores, pró-reitores, políticos).

\section{CONSIDERAÇÕES FINAIS}

A necessidade de uma educação inovadora na graduação vem se justificando pela formação de egressos capazes de atuarem em uma sociedade do conhecimento cada vez mais complexa e fluída. Para isso, se faz necessário o rompimento das atuais dinâmicas de ensino pautada na transmissão de conteúdo, memorização de fatos, nomes, teorias e compartimentalização do conhecimento. Aparentemente, esse seria um problema simples de ser resolvido, uma vez que bastaria ao docente universitário a escolha e implementação por essas mudanças em suas aulas. Na prática, o que se observa 
são docentes que sucumbem às abordagens pedagógicas tradicionais e "seguras", seja pelos altos níveis de estresse e pressão provenientes do próprio ambiente de ensino, das atividades acadêmicas e relações interpessoais ou mesmo pela falta de interesse e apoio institucional.

0 modelo da fragilidade pedagógica reúne, explicita e descreve pontos de tensão do ambiente acadêmico que impedem a inovação do ensino, oferecendo um meio prático de potencializar o desenvolvimento pedagógico e a resiliência na universidade. Apesar de resultados promissores nas mais variadas áreas de conhecimento, o modelo carrega em si uma conotação negativa, que coloca foco nas "reclamações" dos docentes, no "combate" em nível institucional, além da relação que os indivíduos fazem entre fragilidade e "fraqueza".

Neste artigo, foi proposta uma abordagem positiva, humanista e sistêmica de encarar o problema. Focada na promoção da saúde pedagógica, docentes e lideranças acadêmicas precisam trabalhar em conjunto para superar obstáculos de cunho pessoal, profissional, pedagógico e acadêmico que possam impedir atitudes inovadoras. Diferente dos atuais modelos de formação universitária, o modelo pautado na saúde pedagógica é centrado: (1) nas interações da tríade alunosdocentes-sistema; (2) no diálogo aberto e franco que permite aumentar o senso de coerência; (3) no compartilhamento e discussão sobre as diferentes visões de ensino; e, por fim, (4) no engajamento e comprometimento institucional de apoio às práticas de ensino inovadoras.

Para sintetizar as informações apresentadas neste trabalho, um mapa conceitual ${ }^{2}$ foi elaborado para responder à pergunta "Quais as características de um modelo de formação universitária centrado na saúde pedagógica?".

\section{Figura 1 - Mapa conceitual elaborado para sintetizar os principais requisitos para um modelo de formação pedagógica de docentes universitários pautado na promoção da saúde pedagógica.}

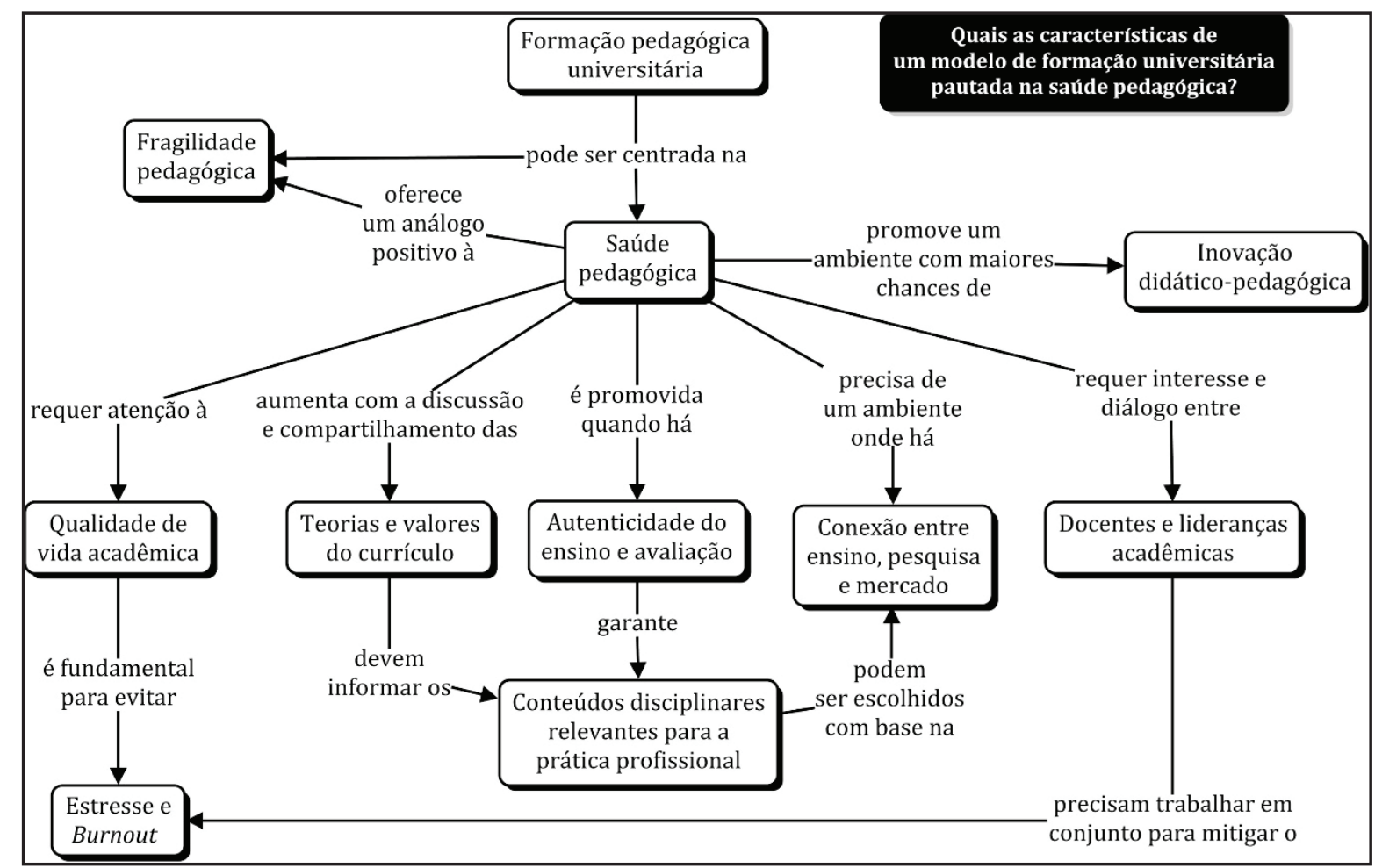

Fonte: A autora.

\section{REFERÊNCIAS}

ADCROFT, Andy. Business. In: KINCHIN, Ian M.; WINSTONE, Naomi E. (eds.) Exploring pedagogic frailty and resilience: case studies of academic narrative. Londres: Brill, 2018. p. 77-90.

2 Mapas conceituais são organizadores gráficos de representação do conhecimento (NOVAK, 2010). Eles são formados por uma rede de proposições, isto é, a união de conceitos por um termo de ligação. Os conceitos são usualmente organizados de modo hierárquico e respondem à uma pergunta focal definida pelo autor do mapa (AGUIAR; CORREIA, 2013). 
AGUIAR Joana G.; CORREIA, Paulo Rogério M. Como fazer bons mapas conceituais? Estabelecendo parâmetros de referências e propondo atividades de treinamento. Revista Brasileira de Pesquisa em Educação em Ciências, v. 13, n. 2, p. 141-157, 2013.

AGUIAR Joana G.; CORREIA, Paulo Rogério M. Um novo olhar sobre a vida acadêmica: estudo de caso sobre as concepções de docentes universitários. Educação e Pesquisa [online], v. 45, p. 1-30, 2019.

ANTONOVSKY, Aaron. Unravelling the mystery of health: how people manage stress and stay well. São Francisco, CA: Jossey Bass, 1987.

AUDY, Jorge Luis Nicolas; MOROSINI, Marília Costa. Inovação e empreendedorismo na universidade. Porto Alegre, RS: Edipucrs, 2006.

BACICH, Lilian; MORAN, José (orgs.). Metodologias ativas para uma educação inovadora: uma abordagem teórico-prática. Porto Alegre, RS: Penso, 2018.

BAILEY, Gillian. Accountability and the rise of "play safe" pedagogical practices. Education + Training, [online], v. 56, n. 7, p. 663-674, 2014.

BARNETT, Ronald. A universidade em uma era de supercomplexidade. São Paulo, SP: Universidade Anhembi Morumbi, 2005.

BATTESTIN, Cláudia; NOGARO, Arnaldo. Sentidos e cotornos da inovação na educação. Holos, v. 2, p. 357-372, 2016.

BEHNEJAD, Alireza. Engineering. In: KINCHIN, Ian M.; WINSTONE, Naomi E. (eds.) Exploring pedagogic frailty and resilience: case studies of academic narrative. Londres: Brill, 2018. p. 35-45.

BENNETT, Sue; HARPER, Barry; HEDBERG, John. Designing real-life cases to support authentic design activities. Australian Journal of Educational Technology, v.18, n.1, p.1-12, 2002.

BORINE, Rita de Cassia Calderani; WANDERLEY, Kátia da Silva; BASSITT, Débora Pastore. Relação entre a qualidade de vida e o estresse em acadêmicos da área da saúde. Estudos Interdisciplinares em Psicologia, v. 6, n. 1, p. 100-118, 2015.

BYRNE, Barbara M. Burnout: investigating the impact of background variables for elementary, intermediate, secondary, and university educators. Teaching \& Teacher Education, v. 7, n. 2, p. 197-209, 1991.

CARBONELL, Jaume. A aventura de inovar: a mudança na escola. Porto Alegre, RS: Artmed Editora, 2002.

CARLOTTO, Mary Sandra. A síndrome de burnout e o trabalho docente. Psicologia em Estudo, v. 7, n. 1, p. 21-29, 2002.

CARLOTTO, Mary Sandra; CÂMARA, Sheila Gonçalves. Preditores da síndrome de burnout em professores. Psicologia Escolar e Educacional, v. 11, n. 1, p. 101-110, 2007.

CLEGG, Sue. Academic identities under threat? British Educational Research Journal, v. 34, n. 3, p. 329-345, 2008.

CORREIA, Paulo Rogério M.; AGUIAR, Joana G.; KINCHIN, Ian M. Mapping pedagogic frailty in science education: a comparison of academics' espoused values. In: BIENNIAL EUROPEAN ASSOCIATION FOR RESEARCH ON LEARNING AND INSTRUCTION (EARLI) CONFERENCE, 17th [Tampere, Finlândia, set. 2017]. Finlândia. Anais [...]. Tampere: Universidade de Tampere, 2017, p. 125.

CUNHA, Maria Isabel. Prática pedagógica e inovação: experiências em foco. In: SEMINÁRIO INOVAÇÃO PEDAGÓGICA: REPENSANDO ESTRATÉGIAS DE FORMAÇÃO ACADÊMICO-PROFISSIONAL EM DIÁLOGO ENTRE EDUCAÇÃO BÁSICA E EDUCAÇÃO SUPERIOR, II [Uruguaiana, RS, 09.11.2018]. Anais [...]. Uruguaiana, RS: Unipampa, 2018. 
DERHAM, Cathrine. Nursing. In: KINCHIN, Ian M.; WINSTONE, Naomi E. (eds.). Exploring pedagogic frailty and resilience: case studies of academic narrative. Londres: Brill, 2018. p. 61-75.

ESTEVES, Manuela. Para a excelência pedagógica do ensino superior. Sísifo: Revista de Ciências da Educação, v. 7, p. 101$110,2008$.

FARIAS, Isabel Maria S. Inovação, mudança e cultura docente. Brasilia, DF: Liber Livro, 2006.

FULLAN, Michael G. El cambio educativo: guía de planeación para maestros. México: Trillas, 2000.

GARCIA, Paulo Sérgio. Inovação e formação contínua de professores de ciências. Educação em Foco, v. 12, n. 13, p. 161189, 2009.

GOERGEN, Pedro. Formação superior: entre o mercado e a cidadania. In: PEREIRA, Elisabete. Universidade e currículo: perspectivas de educação geral. Campinas, SP: Mercado das Letras, 2010. p. 17-40.

HARRISON, Brian J. Are you destined to burn out? Fund Raising Management, v. 30, n. 3, p. 25-28, 1999.

HOSEIN, Anesa. Pedagogic frailty and research-teaching nexus. In: KINCHIN, Ian; WINSTONE, Naomi (eds.). Pedagogic frailty and resilience in the university. Roterdã: Sense, 2017. p. 135-150.

JEUNON, Ester E.; CORREA, Luis Álvaro S.; DUARTE, Leonora C.; GUIMARÃES, Eloisa H. Burnout syndrome: a study with professors at a public university in Maranhão. Revista Gestão \& Tecnologia, v. 17, n. 2, p. 252-268, 2017.

KINCHIN, Ian M. et al. Charting the elements of pedagogic frailty. Educational Research, v. 58, n. 1, p. 1-23, 2016.

KINCHIN, Ian M. Visualising the pedagogic frailty model as a frame for the scholarship of teaching and learning. PSU Research Review, v. 1, n. 3, p. 184-193, 2017.

KINCHIN, Ian M.; FRANCIS, Robert. Mapping pedagogic frailty in geography education: a framed autoethnographic case study. Journal of Geography in Higher Education, v. 41, n. 1, p. 56-74, 2017.

KINCHIN, Ian M.; WILEY, Christopher. Tracing pedagogic frailty in arts and humanities education: an autoethnographic perspective. Arts and Humanities in Higher Education, v. 17, n. 2, p. 241-264, 2018.

KINCHIN, Ian; LYGO-BAKER, Simon; HAY, David. Universities as centres of non-learning. Studies in Higher Education, v. 33, n. 1, p. 89-103, 2008.

KINCHIN, Ian; WINSTONE, Naomi (eds.). Pedagogic frailty and resilience in the university. Roterdã: Sense, 2017.

KUNSCH, Margarida Maria K. Comunicação, inovação, empreendedorismo: um campo aberto para novas pesquisas. Organicom, v. 16, n. 31, p. 11-14, 2019.

LUCARELLI, Elisa. Um desafio institucional: inovação e formação pedagógica do docente universitário. In: CASTANHO, Sergio; CASTANHO, Maria Eugênia L. M. (orgs.). O que há de novo na educação superior: do projeto pedagógico à prática transformadora. Campinas, SP: Papirus, 2000. p. 61-74.

MASLACH, Christina. Burnout: the cost of caring. New Jersey: Prentice Hall, 1982.

MASON, Luke. Law. In: KINCHIN, Ian M.; WINSTONE, Naomi E. (eds.) Exploring pedagogic frailty and resilience: case studies of academic narrative. Londres: Brill, 2018. p. 105-122. 
NILSSON, Marie; EJLERTSSON, Göran; ANDERSSON, Ingemar; BLOMQVIST, Kerstin. Caring as a salutogenic aspect in teachers' lives. Teaching and Teacher Education, v. 46, p. 51-61, 2015.

NOVAK, John D. Learning, creating and using knowledge: concept maps as facilitative tools in schools and corporations. 2nd ed. New York, NY: Routledge, 2010.

ROBERTSON, Jane; BOND, Carol. Experiences of the relation between teaching and research: what do academics values? Higher Education Research \& Development, v. 20, n. 1, p. 5-19, 2001.

SOUSA SANTOS, Boaventura. A universidade no século XXI: para uma reforma democrática e emancipatória da universidade. São Paulo, SP: Cortez, 2010.

TALBOT, Martin. Monkey see, monkey do: a critique of the competency model in graduate medical education. Medical Education, v. 38, n. 6, p. 587-592, 2004.

UNESCO. Towards knowledge societies: Unesco world report. Paris: Unesco, 2005.

USHERWOOD, Simon. Politics. In: KINCHIN, Ian M.; WINSTONE, Naomi E. (eds.) Exploring pedagogic frailty and resilience: case studies of academic narrative. Londres: Brill, 2018. p. 91-103.

WHELLIGAN, Daniel. Chemistry. In: KINCHIN, Ian M.; WINSTONE, Naomi E. (eds.). Exploring pedagogic frailty and resilience: case studies of academic narrative. Londres: Brill, 2018. p. 17-31.

WINSTONE, Naomi E.; KINCHIN, Ian M. Teaching sensitive issues: psychological literacy as an antidote to pedagogic frailty. Psychology Teaching Review, v. 23, n. 1, p. 15-29, 2017.

ZABALZA, Miguel Ángel. O ensino universitário: seu cenário e seus protagonistas. Porto Alegre, RS: Artmed, 2004.

Texto recebido em 06.03.2020 e aprovado em 21.03.2020. 